\title{
Additional Serotypes of Mycobacterium scrofulaceum, Mycobacterium gordonae, Mycobacterium marinum, and Mycobacterium xenopi Determined by Agglutination
}

\author{
SARAH GOSLEE, TAMARA KLEEVE RYNEARSON, AND EMANUEL WOLINSKY \\ Departments of Medicine and Pathology, Case Western Reserve University School of Medicine at Cleveland \\ Metropolitan General Hospital, Cleveland, Ohio 44109
}

\begin{abstract}
Agglutination serotypes were investigated by testing mycobacterial cultures from various sources with a large battery of antisera raised in rabbits. Absorption of agglutinins was performed when necessary to clarify serological relationships. Types in addition to those already described were recognized as follows: Mycobacterium scrofulaceum, serotype Cole; Mycobacterium gordonae, seven serotypes (34 strains isolated from various water sources were serotype Marshall); Mycobacterium marinum types 1 and 2; and Mycobacterium xenopi. It is proposed that these types be numbered, respectively, as follows: Mycobacterium avium complex, serotype 44; $M$. gordonae, serotypes 1 to 7 (Kowal, Marshall, Szent, Puntal, Moore, Lanton, and Brown, respectively); $M$. marinum, serotypes 1 and 2; and $M$. xenopi. The strains of $M$. xenopi included in this study could not be separated into different types by the techniques utilized.
\end{abstract}

Three serotypes of Mycobacterium scrofulaceum are recognized and have been designated as numbers 41,42 , and $43(4,7)$. Preliminary reports on three or four types of Mycobacterium gordonae were presented by Rynearson and Wolinsky in 1967 (Am. Rev. Resp. Dis. 96:155) and in 1970 (Correlation of serological biochemical and clinical aspects of slowly growing mycobacteria; Round Table on "Immunological Approaches to Mycobacterial Taxonomy;" 70th Annu. Meet. Am. Soc. Microbiol., Boston, 1970). Twenty-four strains of Mycobacterium marinum were found to be antigenically homogeneous by agglutination (6), and nothing has been published to our knowledge concerning the antigenic varieties of Mycobacterium xenopi. The purpose of this report is to document the serological relationships which allow us to propose additional serotypes for the mycobacteria of these species.

\section{MATERIALS AND METHODS}

Antiserum production. The mycobacteria used for production of antisera are shown in Table 1 . The strains were grown on Middlebrook $7 \mathrm{H} 10$ agar slants, washed off with phosphate-buffered saline ( $\mathrm{pH} 7.0$ to 7.2), and pipetted into sterile tubes. Large clumps were removed by settling, after which the suspensions were streaked out on $7 \mathrm{H} 10$ agar plates and blood agar plates to assure purity. After heating in a $70 \mathrm{C}$ water bath for $70 \mathrm{~min}$, the suspensions were centrifuged at $2,500 \mathrm{rpm}$ and washed three times with sterile buffer. The cell density was adjusted by a Hopkins tube so that $1 \mathrm{ml}$ of suspension contained $0.01 \mathrm{ml}$ of packed cells, and the suspen- sions were stored in vaccine bottles. Each culture suspension was injected intravenously into two or three 8- to $10-\mathrm{lb}$. (approximately $3.6-$ to $4.5-\mathrm{kg}$ ) albino rabbits. The animals received a series of six injections at 3 - or 4-day intervals, starting with 0.1 $\mathrm{ml}$ and gradually increasing to $1.0 \mathrm{ml}$. A few days after the last injection, the agglutination titer for the homologous culture was determined on a sample of venous blood. One or two further injections were given if the titer was less than 1:640. The rabbits were bled finally from the heart, and the sera were tubed with 1:10,000 merthiolate as preservative and frozen for later use.

Agglutinations. Agglutinations and absorptions were done by the methods of Schaefer $(4,5)$. In addition, twofold dilutions were made in buffered saline to titer the sera with both homologous and heterologous strains. Agglutination was judged after 3 to $4 \mathrm{~h}$ at $37 \mathrm{C}$ and checked after overnight refrigeration. The titer was defined as the highest dilution of serum which produced a definite, although not necessarily complete, agglutination.

Strains were identified at the specific level by the criteria described by Kubica (2). The cultures examined for agglutination either were isolated in this laboratory from patient material, soil, and water, or were stock strains acquired from various sources, as indicated in the tables.

\section{RESULTS}

One new $M$. scrofulaceum serotype was recognized. The results for the four strains of the proposed serotype Cole are shown in Table 2. These strains had the characteristic biochemical and tinctorial reactions of $M$. scrofulaceum. No cross-reactions with any other $M$. scrofula- 


\begin{tabular}{|c|c|c|c|}
\hline Strain no. & Source $^{a}$ & Serotype & Mycobacterial species \\
\hline 356 & P-5, received from Runyon (White, sput, silicosis) & Cole & M. scrofulaceum \\
\hline 357 & P-6, received from Runyon (Cole, sput, lung disease) & Cole & M. scrofulaceum \\
\hline 154 & Kowalczyk, sput, cas, CMGH & Kowal & M. gordonae \\
\hline 144 & Tap water, CMGH & Kowal & M. gordonae \\
\hline 430 & Soil, New Jersey & Puntal & $M$. gordonae \\
\hline 848 & Legeza, sput, cas, CMGH. & Puntal & $M$. gordonae \\
\hline 187 & Szentendrei, tonsil, CMGH & Szent & $M$. gordonae \\
\hline 186 & Pentek, tonsil, CMGH & Szent & $M$. gordonae \\
\hline 46 & Marshall, lung resection, UHC & Marshall & $M$. gordonae \\
\hline 48 & O'Neil, sput, CMGH & Marshall & $M$. gordonae \\
\hline 64 & Wohl, lung resection, UHC & Marshall & M. gordonae \\
\hline 778 & Jolly, gastric, cas, CMGH & Marshall & $M$. gordonae \\
\hline 949 & Lanton, gastric, cas, CMGH & Lanton & $M$. gordonae \\
\hline 617 & Soil, Cleveland, Ohio & Lanton & $M$. gordonae \\
\hline 1083 & Brown, sput, cas, CMGH & Brown & $M$. gordonae \\
\hline 209 & Soil, Shaker Lakes, Cleveland, Ohio & Brown & $M$. gordonae \\
\hline 429 & Soil, yard of Moore, Cleveland, Ohio & Moore & $M$. gordonae \\
\hline 595 & Soil, Cleveland, Ohio & Moore & $M$. gordonae \\
\hline 610 & Soil, Cleveland, Ohio & Moore & M. gordonae \\
\hline 613 & Soil, Cleveland, Ohio & Moore & $M$. gordonae \\
\hline 338 & $\begin{array}{l}\text { Received from Runyon as \#1328; originally from } \\
\text { Schaefer, USPHS \#474 }\end{array}$ & 1 & M. marinum \\
\hline 347 & Received from Runyon as \#2733, ATCC \#11565 & 1 & M. marinum \\
\hline 962 & Fox, olecranon bursa fluid, UHC & 2 & M. marinum \\
\hline 1010 & $\begin{array}{l}\text { Mattingley, skin lesion, Memorial Hospital, Easton, } \\
\text { Md. }\end{array}$ & 2 & M. marinum \\
\hline 1181 & $\begin{array}{l}\text { Serum and culture received from Schaefer: \#931, } \\
\text { Hernandez }\end{array}$ & 1 & M. marinum \\
\hline 1182 & $\begin{array}{l}\text { Serum and culture received from Schaefer: \#661 and } \\
662 \text {, Ellender }{ }^{b}\end{array}$ & 1 & M. marinum \\
\hline 1111 & $\begin{array}{l}\text { Serum and culture received from Schaefer: \#712, } \\
\text { Francingues }\end{array}$ & 2 & M. marinum \\
\hline 1183 & $\begin{array}{l}\text { Serum and culture received from Schaefer: } \# 799 \text {, } \\
\text { Geraci }^{b}\end{array}$ & 2 & M. marinum \\
\hline 336 & $\begin{array}{l}\text { Received from Runyon as \#1162; originally from } \\
\text { Schaefer }\end{array}$ & $?$ & $M$. marinum \\
\hline 344 & $\begin{array}{l}\text { Received from Runyon as \#2337; knee lesion strain } \\
\text { recovered from mouse }\end{array}$ & $?$ & M. marinum \\
\hline 871 & Ebenger, hand abscess, CMGH & $?$ & M. marinum \\
\hline 351 & NCTC \#10042 (type strain) & & M. xenopi \\
\hline 668 & Soil, Montana, received from Runyon as $\# 3244$ & & M. xenopi \\
\hline 1174 & $\begin{array}{l}\text { Serum and culture received from Schaefer: Cardiff, } \\
\# 25592^{b}\end{array}$ & & M. xenopi \\
\hline 1173 & $\begin{array}{l}\text { Serum and culture received from Schaefer; originally } \\
\text { from J. Hawkins, \#72-1097 }\end{array}$ & & M. xenopi \\
\hline
\end{tabular}

a Abbreviations: sput, sputum; cas, casual isolate, defined as a strain isolated from human material once or twice, but not significant in causing disease; CMGH, Cleveland Metropolitan General Hospital; UHC, University Hospitals of Cleveland; USPHS, U.S. Public Health Service.

${ }^{b}$ These materials were provided by the U.S.-Japan Cooperative Medical Science Program, NIAID, through W. B. Schaefer.

TABLE 2. Agglutination titers with proposed Cole serotype of $M$. scrofulaceum

\begin{tabular}{|c|c|c|c|}
\hline \multirow{2}{*}{$\begin{array}{l}\text { Strain } \\
\text { no. }\end{array}$} & \multirow{2}{*}{ Source } & \multicolumn{2}{|c|}{$\begin{array}{c}\text { Agglutination titer to } \\
\text { antiserum: }\end{array}$} \\
\hline & & 356 & 357 \\
\hline 357 & $\begin{array}{l}\text { Cole, sputum, Run- } \\
\text { yon P-6 }\end{array}$ & $1: 320$ & $\geqslant 1: 1,280$ \\
\hline 28 & Cole, sputum ${ }^{a}$ & $1: 320$ & $1: 640$ \\
\hline 356 & $\begin{array}{l}\text { White, sputum, } \\
\text { Runyon P-5 }\end{array}$ & $1: 640$ & $\geqslant 1: 1,280$ \\
\hline $\begin{array}{l}\text { ATCC } \\
23406\end{array}$ & IWGMT study ${ }^{b}$ & $1: 640$ & $1: 2,560$ \\
\hline $161-4$ & $\begin{array}{l}\text { Fish tank, Cleve- } \\
\text { land, Ohio, Aquar- } \\
\text { ium }\end{array}$ & $1: 320$ & $1: 320$ \\
\hline
\end{tabular}

${ }^{a}$ Our stock culture from same source as culture P-6 (patient at Trudeau Sanatorium).

${ }^{b}$ International Working Group on Mycobacterial Taxonomy; received as part of a cooperative study.
TABLE 3. Agglutination titers with proposed Kowal serotype of $M$. gordonae

\begin{tabular}{|c|c|c|c|}
\hline \multirow{2}{*}{$\begin{array}{l}\text { Strain } \\
\text { no. }\end{array}$} & \multirow{2}{*}{ Source $^{a}$} & \multicolumn{2}{|c|}{$\begin{array}{c}\text { Agglutination titer to } \\
\text { antiserum: }\end{array}$} \\
\hline & & 144 & 154 \\
\hline 154 & $\begin{array}{l}\text { Kowalczyk, sputum, } \\
\text { CMGH }\end{array}$ & $>1: 1,280$ & $\geqslant 1: 2,560$ \\
\hline 144 & Tap water, CMGH & $>1: 2,560$ & $>1: 2,560$ \\
\hline 360 & $\begin{array}{l}\text { ATCC } 14470 \text { (type } \\
\text { culture), Runyon } \\
\text { P } 15\end{array}$ & $\geqslant 1: 2,560$ & $1: 1,280$ \\
\hline $\begin{array}{l}\text { ATCC } \\
23398\end{array}$ & $\begin{array}{l}\text { IWGMT }^{b} \text { study, tap } \\
\text { water }\end{array}$ & $1: 1,280$ & $1: 1,280$ \\
\hline $\begin{array}{l}\text { ATCC } \\
23399\end{array}$ & IWGMT study & $1: 2,560$ & $1: 2,560$ \\
\hline 997 & $\begin{array}{l}\text { Jones, sputum, } \\
\text { CMGH }\end{array}$ & $>1: 1,280$ & $1: 640$ \\
\hline
\end{tabular}

${ }^{a}$ For abbreviations, see footnote $a$, Table 1.

${ }^{b}$ See footnote $b$ of Table 2 . 
TABLE 4. Agglutination titers with proposed Marshall serotype of M. gordonae

\begin{tabular}{|c|c|c|c|c|c|}
\hline \multirow{2}{*}{$\begin{array}{c}\text { Strain } \\
\text { no. }\end{array}$} & \multirow{2}{*}{ Source $^{a}$} & \multicolumn{4}{|c|}{ Agglutination titer to antiserum: } \\
\hline & & 46 & 64 & 48 & 778 \\
\hline 46 & Marshall, lung resection, UHC & $1: 640$ & $1: 320$ & $\geqslant 1: 2,560$ & $1: 1,280$ \\
\hline 64 & Wohl, lung resection, UHC & $1: 320$ & $1: 160$ & $1: 1,280$ & $1: 320$ \\
\hline 48 & O'Neil, sputum, CMGH & $1: 320$ & $1: 160$ & $\geqslant 1: 1,280$ & $1: 1,280$ \\
\hline 778 & Jolly, gastric, CMGH & $1: 160$ & $<1: 80$ & $\geqslant 1: 2,560$ & $>1: 2,560$ \\
\hline 4 & Crawley, sputum, CMGH & $1: 1,280$ & $1: 640$ & $\geqslant 1: 2,560$ & $1: 2,560$ \\
\hline 1060 & Media contaminant, Bacty Lab, CMGH & $1: 1,280$ & $1: 1,280$ & $1: 1,280$ & $1: 1,280$ \\
\hline 1064 & Ruttencutter, sputum, CMGH & $1: 160$ & $1: 320$ & $1: 1,280$ & $1: 320$ \\
\hline 34 & $\begin{array}{l}\text { Strains recovered from various water } \\
\text { sources: taps, drinking fountains, zoo, } \\
\text { and aquarium }{ }^{b}\end{array}$ & & & $1: 1,280$ & $1: 1,280$ \\
\hline
\end{tabular}

${ }^{a}$ For abbreviations, see footnote $a$, Table 1 .

${ }^{\circ}$ Goslee and Wolinsky, Am. Rev. Resp. Dis., in press. Once the four type strains were established as one serotype, only the \#48 and \#778 sera were used to agglutinate the water strains.

ceum serotypes were observed, nor were there any significant cross-reactions with other species of mycobacteria or their antisera.

Seven $M$. gordonae serotypes were recognized, as depicted in Tables 3 to 9. Their agglutinations were all clear-cut, and no significant cross-reactions among these serotypes were observed. A cross-reaction between serotype Marshall and Mycobacterium avium complex serotype 4 was seen, but absorption of the type 4

TABLE 5. Agglutination titers with proposed Szent serotype of.M. gordonae

\begin{tabular}{|c|c|c|c|}
\hline \multirow{2}{*}{$\begin{array}{l}\text { Strain } \\
\text { no. }\end{array}$} & \multirow{2}{*}{ Source $^{a}$} & \multicolumn{2}{|c|}{$\begin{array}{c}\text { Agglutination titer to } \\
\text { antiserum: }\end{array}$} \\
\hline & & 186 & 187 \\
\hline 187 & $\begin{array}{l}\text { Szentendrei, tonsil, } \\
\text { CMGH }\end{array}$ & $>1: 640$ & $>1: 1,280$ \\
\hline 138 & $\begin{array}{l}\text { Grady, sputum, } \\
\text { CMGH }\end{array}$ & $>1: 1,280$ & $>1: 1,280$ \\
\hline 186 & $\begin{array}{l}\text { Pentek, tonsil, } \\
\text { CMGH }\end{array}$ & $>1: 1,280$ & $>1: 1,280$ \\
\hline 281 & $\begin{array}{l}\text { Soil, Cleveland, } \\
\text { Ohio }\end{array}$ & $\geqslant 1: 1,280$ & $>1: 640$ \\
\hline 567 & $\begin{array}{l}\text { Soil, Cleveland, } \\
\text { Ohio }\end{array}$ & $>1: 640$ & $>1: 640$ \\
\hline 569 & $\begin{array}{l}\text { Soil, Cleveland, } \\
\text { Ohio }\end{array}$ & $>1: 640$ & $>1: 640$ \\
\hline 679 & $\begin{array}{l}\text { Croff, lymph node, } \\
\text { CMGH }\end{array}$ & $1: 2,560$ & $>1: 2,560$ \\
\hline 781 & $\begin{array}{l}\text { Payne, gastric, } \\
\text { CMGH }\end{array}$ & $1: 1,280$ & $1: 1,280$ \\
\hline 956 & $\begin{array}{l}\text { Oster, sputum, } \\
\text { CMGH }\end{array}$ & $>1: 1,280$ & $>1: 1,280$ \\
\hline $\begin{array}{l}\text { ATCC } \\
23285\end{array}$ & IWGMT $^{b}$ study & $>1: 2,560$ & $>1: 2,560$ \\
\hline $\begin{array}{l}\text { ATCC } \\
\quad 23415\end{array}$ & IWGMT study & $>1: 1,280$ & $>1: 1,280$ \\
\hline $25-2$ & $\begin{array}{l}\text { Water, Niagara } \\
\text { River }\end{array}$ & $1: 640$ & $1: 1,280$ \\
\hline $73-1$ & $\begin{array}{l}\text { Water, kitchen tap, } \\
\text { Oxford, Ohio }\end{array}$ & $1: 640$ & $\geqslant 1: 2,560$ \\
\hline
\end{tabular}

"For abbreviations, see footnote $a$, Table 1.

- See footnote $b$, Table 2 .
TABLE 6. Agglutination titers with proposed Puntal serotype of $M$. gordonae

\begin{tabular}{|c|c|c|c|}
\hline \multirow{2}{*}{$\begin{array}{l}\text { Strain } \\
\text { no. }\end{array}$} & \multirow{2}{*}{ Source $^{a}$} & \multicolumn{2}{|c|}{$\begin{array}{l}\text { Agglutination titer to } \\
\text { antiserum: }\end{array}$} \\
\hline & & 430 & 848 \\
\hline 50 & $\begin{array}{c}\text { Puntal, } \\
\text { CMGH }\end{array}$ & $1: 640-1,280$ & $1: 1,280$ \\
\hline 40 & $\begin{array}{l}\text { Galbreath, sputum, } \\
\text { CMGH }\end{array}$ & $1: 2,560$ & $1: 2,560$ \\
\hline 74 & $\begin{array}{r}\text { Swanson, lung, } \\
\text { UHC }\end{array}$ & $1: 2,560$ & $1: 1,280$ \\
\hline 430 & Soil, New Jersey & $1: 2,560$ & $1: 640$ \\
\hline 565 & Soil & $1: 1,280$ & $1: 2,560$ \\
\hline 848 & $\begin{array}{c}\text { Legeza, sputum, } \\
\text { CMGH }\end{array}$ & $1: 1,280$ & $1: 5,120$ \\
\hline \multirow[t]{4}{*}{999} & $\begin{array}{l}\text { Leckwatch, } \\
\text { tum }^{b}\end{array}$ & & \\
\hline & $5 / 15 / 72$ & $1: 1,280$ & $1: 1,280$ \\
\hline & $8 / 2 / 72$ & $1: 1,280$ & $1: 640$ \\
\hline & $11 / 3 / 72$ & $1: 1,280$ & $1: 320-640$ \\
\hline \multirow[t]{4}{*}{1077} & $\begin{array}{l}\text { Leckwatch, } \\
\text { tum }^{b}\end{array}$ & & \\
\hline & $4 / 18 / 73$ & $1: 2,560$ & $1: 1,280$ \\
\hline & $7 / 15 / 73$ & $1: 2,560$ & $1: 1,280$ \\
\hline & $9 / 17 / 73$ & $1: 1,280$ & $1: 640$ \\
\hline
\end{tabular}

${ }^{a}$ For abbreviations, see footnote $a$, Table 1.

${ }^{b}$ Repeated isolations, probably representing tracheobronchial colonization rather than disease association, from a patient at $\mathrm{CMGH}$ and Cuyahoga County Clinic.

serum with Marshall strains produced a negligible reduction in the type 4 homologous titer. It is noteworthy that the four Brown serotype cultures had an optimum growth temperature of 30 to $34 \mathrm{C}$.

The $M$. marinum strains could be divided into two agglutination groups. The results for type 1 are given in Table 10. It may be seen that a uniform titer of agglutination was not achieved by the five typing sera, including two made in this laboratory and three supplied by Schaefer. Indeed, even repeat tests with the same strain and serum sometimes gave discrep- 
TABLE 7. Agglutination titers with proposed Moore serotype of M. gordonae

\begin{tabular}{|c|c|c|c|c|c|}
\hline \multirow{2}{*}{$\begin{array}{l}\text { Strain } \\
\text { no. }\end{array}$} & \multirow{2}{*}{ Source } & \multicolumn{4}{|c|}{ Agglutination titer to antiserum: } \\
\hline & & 429 & 610 & 595 & 613 \\
\hline $429^{a}$ & $\begin{array}{l}\text { Soil, Moore, Cleveland, } \\
\text { Ohio }\end{array}$ & $>1: 2,560$ & & & \\
\hline $610^{a}$ & Soil, Cleveland, Ohio & $1: 1,280$ & $>1: 1,280$ & & \\
\hline 595 & Soil, Cleveland, Ohio & $1: 2,560$ & $1: 1,280$ & $1: 1,280$ & $\geqslant 1: 5,120$ \\
\hline 613 & Soil, Cleveland, Ohio & $1: 2,560$ & $1: 640$ & $1: 640$ & $1: 1,280$ \\
\hline 616 & Soil, Cleveland, Ohio & $\geqslant 1: 5,120$ & $1: 2,560$ & $1: 1,280$ & $\geqslant 1: 5,120$ \\
\hline 634 & Soil, Cleveland, Ohio & $1: 1,280$ & $1: 1,280$ & $1: 5,120$ & $1: 5,120$ \\
\hline
\end{tabular}

${ }^{a}$ These cultures were lost before other antisera were made.

TABLE 8. Agglutination titers with proposed Lanton serotype of $M$. gordonae

\begin{tabular}{|c|c|c|c|}
\hline \multirow{2}{*}{$\begin{array}{l}\text { Strain } \\
\text { no. }\end{array}$} & \multirow{2}{*}{ Source $^{a}$} & \multicolumn{2}{|c|}{$\begin{array}{c}\text { Agglutination titer to } \\
\text { antiserum: }\end{array}$} \\
\hline & & 617 & 949 \\
\hline 949 & $\begin{array}{l}\text { Lanton, gastric, } \\
\text { CMGH }\end{array}$ & $\geqslant 1: 640$ & $1: 640$ \\
\hline 617 & $\begin{array}{l}\text { Soil, Cleveland, } \\
\text { Ohio }\end{array}$ & $1: 640$ & $1: 320$ \\
\hline 998 & $\begin{array}{l}\text { Lanton, sputum, } \\
\text { Cuyahoga } \\
\text { County Clinic }\end{array}$ & $1: 1,280$ & $1: 1,280$ \\
\hline $87-1$ & $\begin{array}{l}\text { Water, drinking } \\
\text { fountain, } \\
\text { Cleveland, Ohio } \\
\text { (liter sample) }\end{array}$ & $\geqslant 1: 2,560$ & $\geqslant 1: 2,560$ \\
\hline $87-2^{b}$ & $\begin{array}{l}\text { Water, drinking } \\
\text { fountain, } \\
\text { Cleveland, Ohio } \\
\text { (liter sample) }\end{array}$ & $\geqslant 1: 1,280$ & $\geqslant 1: 2,560$ \\
\hline $87-3^{b}$ & $\begin{array}{l}\text { Water, drinking } \\
\text { fountain, } \\
\text { Cleveland, Ohio } \\
\text { (liter sample) }\end{array}$ & $1: 640$ & $1: 2,560$ \\
\hline $88-1$ & $\begin{array}{l}\text { Water, drinking } \\
\text { fountain, } \\
\text { Cleveland, Ohio } \\
\text { (swab sample) }\end{array}$ & $1: 2,560$ & $1: 2,560$ \\
\hline $88-2^{b}$ & $\begin{array}{l}\text { Water, drinking } \\
\text { fountain, } \\
\text { Cleveland, Ohio } \\
\text { (swab sample) }\end{array}$ & $\geqslant 1: 2,560$ & $\geqslant 1: 2,560$ \\
\hline $88-3^{b}$ & $\begin{array}{l}\text { Water, drinking } \\
\text { fountain, } \\
\text { Cleveland, Ohio } \\
\text { (swab sample) }\end{array}$ & $\geqslant 1: 2,560$ & $\geqslant 1: 2,560$ \\
\hline $88-4^{b}$ & $\begin{array}{l}\text { Water, drinking } \\
\text { fountain, } \\
\text { Cleveland, Ohio } \\
\text { (swab sample) }\end{array}$ & $\geqslant 1: 2,560$ & $\geqslant 1: 2,560$ \\
\hline
\end{tabular}

${ }^{a}$ For abbreviations, see footnote $a$, Table 1 .

${ }^{b}$ Different colonies from original isolation media.

ant results. Similar inconsistencies were noted with sera made from three other strains, numbers 336,344 , and 871 .

The reactions of $M$. marinum type 2 are presented in Table 11. Only one of the strains reacted strongly with all four type 2 sera, but 1010 and 1111 were not agglutinated by Schaefer's serum 799. Culture 1183 tended to autoag-
TABLE 9. Agglutination titers with proposed Brown serotype $^{a}$ of $M$. gordonae

\begin{tabular}{cccc}
\hline \multirow{2}{*}{$\begin{array}{c}\text { Strain } \\
\text { no. }\end{array}$} & \multicolumn{1}{c}{ Source $^{b}$} & \multicolumn{2}{c}{$\begin{array}{c}\text { Agglutination titer to } \\
\text { antiserum: }\end{array}$} \\
\cline { 2 - 4 } 1083 & $\begin{array}{c}\text { Brown, sputum, } \\
\text { CMGH (5/26/73) }\end{array}$ & $\geqslant 1: 1,280$ & $\geqslant 1: 1,280$ \\
1084 & $\begin{array}{c}\text { Brown, sputum, } \\
\text { CMGH (5/24/75) }\end{array}$ & $1: 1,280$ & $1: 1,280$ \\
209 & $\begin{array}{c}\text { Soil, Shaker Lakes, } \\
\text { Cleveland, Ohio } \\
\text { Leindecker, gastric, } \\
\text { Euclid General } \\
\text { Hospital, } \\
\text { Cleveland, Ohio }\end{array}$ & $1: 1,280$ & $1: 1,280$ \\
& - & & \\
\hline
\end{tabular}

${ }^{a}$ All strains in this serotype had an optimum growth temperature of 30 to $34 \mathrm{C}$.

- For abbreviations, see footnote $a$, Table 1.

glutinate upon repeated subculture, and no titer could be obtained with our type 2 serum pair. Cross-absorptions between type 1 and 2 cultures and sera were performed. The type 2 strains rarely reacted with type 1 sera, and when this occurred the cross-reacting antibody was easily absorbed out with only a small reduction in homologous titer. Similarly, absorption of our type 2 serum 962 with our type 1 strain 338 resulted in no reduction of the homologous titer.

Table 12 shows the serological reactions of the $10 \mathrm{M}$. xenopi strains in our collection which could be suspended smoothly without spontaneous agglutination. For the most part, these strains reacted equally with all four sera (our pair and Schaefer's pair). Absorption of agglutinins was performed with an additional seven rough strains which were not suitable for agglutination, and the results of a representative set of absorptions are shown in Table 13. The two strains from which our serum pair was derived $(351,668)$ removed the $M$. xenopi agglutinins from their own sera and did not agglutinate significantly with Schaefer's homologously absorbed sera. A similar relationship was found between Schaefer's two strains and our serum 
TABLE 10. Agglutination titers with proposed serotype 1 of $M$. marinum

\begin{tabular}{|c|c|c|c|c|c|c|c|c|c|}
\hline \multirow{2}{*}{$\begin{array}{l}\text { Strain } \\
\text { no. }\end{array}$} & \multirow{2}{*}{ Source $^{a}$} & \multicolumn{8}{|c|}{ Agglutination titer to antiserum: } \\
\hline & & 338 & 347 & $931^{b}$ & $661^{b}$ & $662^{\circ}$ & 336 & 344 & 871 \\
\hline 338 & $\begin{array}{l}\text { Our serotype } \\
\text { strain, Runyon } \\
1328\end{array}$ & $\geqslant 1: 1,280$ & $1: 80$ & $\geqslant 1: 640$ & $1: 320$ & $1: 320$ & $1: 80$ & $1: 320$ & $1: 40$ \\
\hline 347 & $\begin{array}{l}\text { Our serotype } \\
\text { strain, Runyon } \\
2733\end{array}$ & $\geqslant 1: 2,560$ & $1: 320$ & $\geqslant 1: 640$ & $\geqslant 1: 640$ & $\geqslant 1: 640$ & $1: 640$ & $1: 1,280$ & $1: 320$ \\
\hline 336 & Runyon 1162 & $\geqslant 1: 1,280$ & $\mathrm{Nil}^{c}$ & $\geqslant 1: 640$ & $1: 320$ & Nil & $\begin{array}{c}1: 160 \\
\geqslant 1: 1,280^{d}\end{array}$ & 1:80 & Nil \\
\hline 344 & Runyon 2337 & $\geqslant 1: 1,280$ & $\begin{array}{l}\mathrm{Nil} \\
1: 640\end{array}$ & $\geqslant 1: 640$ & $\geqslant 1: 640$ & $\geqslant 1: 640$ & $1: 160$ & $\begin{aligned} \geqslant & 1: 1,280 \\
& 1: 320\end{aligned}$ & $1: 160$ \\
\hline 871 & $\begin{array}{l}\text { Ebenger, hand } \\
\text { abscess, CMGH }\end{array}$ & $\geqslant 1: 2,560$ & $\geqslant 1: 2,560$ & $1: 320$ & 1:160 & Nil & $1: 640$ & $1: 1,280$ & $\geqslant 1: 1,280$ \\
\hline 341 & $\begin{array}{l}\text { Runyon } 2312, \\
\text { originally from } \\
\text { W. F. Shinn } \\
\text { (Magnusson 172) }\end{array}$ & $\begin{array}{l}1: 320 \\
1: 1,280\end{array}$ & $\begin{array}{l}1: 80 \\
1: 80 \\
1: 160\end{array}$ & $1: 640$ & $1: 640$ & Nil & $1: 320$ & $1: 320$ & Nil \\
\hline 928 & $\begin{array}{l}\text { Ellender, } \\
\text { Schaefer's } 661 \\
\text { and } 662^{b}\end{array}$ & $\geqslant 1: 1,280$ & $\geqslant 1: 640$ & $\geqslant 1: 640$ & $\geqslant 1: 640$ & $\geqslant 1: 640$ & $\geqslant 1: 1,280$ & $\geqslant 1: 1,280$ & $\geqslant 1: 1,280$ \\
\hline 1052 & $\begin{array}{l}\text { Russo, scratch from } \\
\text { aquarium, } \\
\text { University } \\
\text { Hospital, } \\
\text { Cleveland, Ohio }\end{array}$ & $1: 640$ & $\begin{array}{l}\geqslant 1: 1,280 \\
\text { Nil }\end{array}$ & $\geqslant 1: 640$ & $\geqslant 1: 640$ & $1: 320$ & $1: 320$ & $1: 160$ & $\geqslant 1: 1,280$ \\
\hline 1181 & $\begin{array}{l}\text { Hernandez, } \\
\text { Schaefer's } 931^{\circ}\end{array}$ & $\geqslant 1: 1,280$ & $1: 80$ & $\geqslant 1: 640$ & $1: 160$ & Nil & $1: 640$ & $1: 320$ & $1: 320$ \\
\hline
\end{tabular}

${ }^{a}$ For abbreviations, see footnote $a$, Table 1 .

${ }^{b}$ Provided by the U.S.-Japan Cooperative Medical Science Program, NIAID, through W. B. Schaefer.

c Designates a titer of less than 1:40.

${ }^{d}$ Two figures indicate different titers obtained on repeat tests.

TABLE 11. Agglutination titers with proposed serotype 2 of $M$. marinum

\begin{tabular}{|c|c|c|c|c|c|}
\hline \multirow{3}{*}{$\begin{array}{c}\text { Strain } \\
\text { no. }\end{array}$} & \multirow{3}{*}{ Source ${ }^{a}$} & \multicolumn{4}{|c|}{ Agglutination titer to: } \\
\hline & & \multicolumn{2}{|c|}{ Our sera } & \multicolumn{2}{|c|}{ Schaefer's sera ${ }^{b}$} \\
\hline & & 962 & 1010 & 712 & 799 \\
\hline 962 & Fox, bursa, UHC & $\geqslant 1: 1,280$ & $\geqslant 1: 1,280$ & $\geqslant 1: 640$ & $\geqslant 1: 640$ \\
\hline 1010 & Mattingley, skin, Maryland & $\geqslant 1: 1,280$ & $1: 320$ & $\geqslant 1: 640$ & $\mathrm{Nil}^{c}$ \\
\hline 1111 & Francinques, Schaefer's $712^{b}$ & $\geqslant 1: 1,280$ & $1: 640$ & $\geqslant 1: 640$ & $\mathrm{Nil}$ \\
\hline 1183 & Geraci, Schaefer's $799^{b}$ & $-d$ & $-{ }^{d}$ & $1: 640$ & $1: 1,280$ \\
\hline
\end{tabular}

${ }^{a}$ For abbreviations, see footnote $a$, Table 1.

${ }^{b}$ Provided by the U.S.-Japan Cooperative Medical Science Program, NIAID.

c Designates a titer of less than 1:40.

${ }^{a}$ Culture autoagglutinated.

pair. The "unknown" strain 1155 removed all agglutinins from Schaefer's sera, but it absorbed relatively little of the agglutinins from our 351 serum (usually a twofold drop in homologous titer on repeated testing) and only slightly more (fourfold drop) from our 668 serum. The other six rough strains reacted in a similar fashion.

\section{DISCUSSION}

A fourth serotype of $M$. scrofulaceum, serotype Cole, has been proposed. Of the five strains in this group, two originated from the same patient, although they arrived in the collection by different routes. These strains and the White strain (356) were considered to be human pathogens. ATCC 23406 was received as part of an international cooperative study, and its association with human disease is uncertain. One strain was recovered from aquarium water.

$M$. gordonae is an environmental saprophyte which is not usually associated with disease in man. Gunthorpe and Stanford (1) found five species-specific antigens by immunodiffusion, and Pattyn et al. (3) studied over 30 strains, 
TABLE 12. Agglutination titers with proposed M. xenopi serotype

\begin{tabular}{|c|c|c|c|c|c|}
\hline \multirow{3}{*}{$\begin{array}{l}\text { Strain } \\
\text { no. }\end{array}$} & \multirow{3}{*}{ Source $^{a}$} & \multicolumn{4}{|c|}{ Agglutination titer to: } \\
\hline & & \multicolumn{2}{|c|}{ Our sera } & \multicolumn{2}{|c|}{ Schaefer's sera ${ }^{b}$} \\
\hline & & 351 & 668 & 927 & 907 \\
\hline 351 & NCTC 10042 (type strain) & $1: 640$ & $1: 1,280$ & $1: 1,280$ & $1: 640$ \\
\hline 668 & Runyon: Montana 3244 & $1: 320$ & $1: 1,280$ & $1: 320$ & $1: 160$ \\
\hline 669 & Runyon: Arkansas 3254 & $1: 320$ & $1: 320$ & $1: 320$ & $1: 160$ \\
\hline 967 & Keyerleber, sputum, CMGH & $1: 640$ & $1: 320$ & $1: 320$ & $1: 320$ \\
\hline 1127 & $\begin{array}{l}\text { Hawkins: water, 74-0202, West Haven, } \\
\text { Conn. }\end{array}$ & $1: 640$ & $1: 640$ & $1: 1,280$ & $1: 1,280$ \\
\hline 1130 & Hawkins: Faucher, sputum, 74-0704 & $1: 320$ & $1: 640$ & $1: 1,280$ & $1: 1,280$ \\
\hline 1131 & Hawkins: Faucher, sputum, 74-0781 & $1: 320$ & $1: 640$ & $1: 640$ & $1: 640$ \\
\hline 1132 & Hawkins: MacNevin, sputum, 74-0782 & $1: 320$ & $1: 640$ & $1: 1,280$ & $1: 1,280$ \\
\hline 1173 & $\begin{array}{l}\text { Schaefer's 927: originally from Hawkins 72- } \\
1097\end{array}$ & $1: 640$ & $1: 640$ & $1: 640$ & $1: 320$ \\
\hline 1174 & Schaefer's 907: Cardiff 25592 & $1: 640$ & $1: 640$ & $1: 80$ & $1: 160$ \\
\hline
\end{tabular}

"For abbreviations, see footnote $a$, Table 1 .

${ }^{b}$ Provided by the U.S.-Japan Cooperative Medical Science Program, NIAID.

TABLE 13. M. xenopi: results of absorptions with homologous strain and with strain $1155^{a}$ of our serum pair $(351,668)$ and Schaefer's serum pair $(907,927)^{b}$

\begin{tabular}{|c|c|c|c|c|c|c|c|c|c|c|c|c|}
\hline \multirow{3}{*}{$\begin{array}{r}\text { Strain } \\
\text { no. }\end{array}$} & \multirow{3}{*}{ Source } & \multicolumn{11}{|c|}{ Agglutination titer to antiserum: } \\
\hline & & $351^{\circ}$ & 351 & 508 & 668 & 668 & ดา7 & 927 & 927 & & 907 & 907 \\
\hline & & 351 & 1155 & 000 & 668 & 1155 & 521 & $\overline{1173}$ & 1155 & Sol & $\overline{1174}$ & $\overline{1155}$ \\
\hline 351 & NCTC 10042 & $\mathrm{Nil}^{d}$ & $1: 320$ & $1: 80$ & Nil & - & $1: 80$ & Nil & _- & $1: 320$ & Nil & - \\
\hline 668 & Runyon: Montana 3244 & Nil & - & $1: 320$ & Nil & $1: 80$ & $1: 320$ & Nil & - & $1: 160$ & Nil & - \\
\hline 1173 & Schaefer's $927^{b}$ & Nil & - & $1: 640$ & Nil & - & $1: 640$ & Nil & $\mathrm{Nil}$ & $1: 320$ & Nil & - \\
\hline 1174 & Schaefer's $907^{b}$ & Nil & - & 1:640 & $\mathrm{Nil}$ & - & $1: 80$ & Nil & - & $1: 160$ & Nil & $\mathrm{Nil}$ \\
\hline
\end{tabular}

" Received from Hawkins, West Haven, Conn.

${ }^{b}$ Provided by the U.S.-Japan Cooperative Medical Science Program, NIAID.

c Indicates serum 351 absorbed with strain 351.

${ }^{d}$ Designates a titer of less than 1:40.

- Agglutination not done.

mostly recovered as laboratory contaminants, by agglutination with four antisera, but neither group of investigators separated the species into serotypes. The results presented herein indicate that at least seven agglutination types may be recognized. No single serotype or group of serotypes contained a larger proportion than the others of the human-derived strains. Most of the $M$. gordonae strains in our collection were not typable with the seven serum pairs (unpublished observations), but the majority of the typable strains were of the Marshall serotype. In particular, of $80 \mathrm{M}$. gordonae strains recovered from water, 34 were serotype Marshall, 34 were not typable with the sera used, and the remaining 12 were distributed among the other six types (Showalter and Wolinsky, submitted for publication). Despite its evident antigenic heterogeneity, it may be possible to delineate additional serotypes of $M$. gordonae by further investigation.

Two types of $M$. marinum, 1 and 2, were separated clearly by absorptions, but several
M. marinum strains were agglutinated by only one of the pair of our as well as of Schaefer's type sera. Three of these strains were selected as possible representatives of a third type. After making antisera to these strains, it was found that the questionable strains and their antisera were closely related, but probably not identical, to type 1 . Further investigation will be necessary to clarify these relationships.

The agglutinations of $M$. xenopi gave somewhat conflicting results. Absorptions indicated that our type strains and Schaefer's type strains were antigenically homogeneous. Similarly, the "unknown" strains absorbed most of the agglutinins from Schaefer's sera. With our sera, however, these strains absorbed relatively little agglutinating activity, causing a drop in homologous titer no greater than fourfold. Thus, it was not possible by the techniques used to clearly recognize more than one type among the $M$. xenopi strains included in this study.

The results with $M$. marinum and $M$.xenopi 
emphasize the importance of using at least a pair of antisera to establish the serotype of an unknown strain, as recommended by Schaefer (4). It may be desirable to use a trio of typing sera for certain more broadly reactive groups.

The proposed new serotypes could be included in the suggested numbering scheme (7) as follows: $M$. avium complex, serotype 44 ( $M$. scrofulaceum, Cole); $M$. gordonae, serotypes 1 to 7 (Kowal, Marshall, Szent, Puntal, Moore, Lanton, and Brown, respectively); $M$. marinum, serotypes 1 and 2; and $M$. xenopi.

\section{ACKNOWLEDGMENTS}

We would like to thank Bruce Elliot, Jr., John Ratz, Bernadette McMahon, David Dodson, and Hollis Shaw for their help in antiserum production and serotyping.

These investigations were supported by grants from the Ohio Lung Association and the Northern Ohio Lung Association.

\section{REPRINT REQUESTS}

Address reprint requests to: Dr. E. Wolinsky, Cleveland Metropolitan General Hospital, 3395 Scranton Road, Cleveland, Ohio 44109 . .

\section{LITERATURE CITED}

1. Gunthorpe, W. J., and J. L. Stanford. 1972. A study of Mycobacterium gordonae and Mycobacterium marianum (scrofulaceum). Br. J. Exp. Pathol, 53:665671.

2. Kubica, G. P. 1973. Differential identification of mycobacteria. VII. Key features for identification of clinically significant mycobacteria. Am. Rev. Resp. Dis. 107:9-21.

3. Pattyn, S. R., M. T. Hermans-Boveroulle, and J. van Ermengem. 1968. A study on slow growing chromogenic (Runyon's Group II) mycobacteria. Zentralbl. Bakteriol. Parasitenk. Infektionskr. Hyg. Abt. I Orig. 207:509-516.

4. Schaefer, W. B. 1965. Serologic identification and classification of the atypical mycobacterial by their agglutination. Am. Rev. Resp. Dis. 92(Suppl.):85-93.

5. Schaefer, W. B. 1967. Type-specificity of atypical mycobacteria in agglutination and antibody absorption tests. Am. Rev. Resp. Dis. 96:1165-1168.

6. Schaefer, W. B., and C. L. Davis. 1961. A bacteriologic and histopathologic study of skin granuloma due to Mycobacterium balnei. Am. Rev. Resp. Dis. 84:837844.

7. Wolinsky, E., and W. B. Schaefer. 1973. Proposed numbering scheme for mycobacterial serotypes by agglutination. Int. J. Syst. Bacteriol. 23:182-183. 